PROCEEDINGS OF THE

AMERICAN MATHEMATICAL SOCIETY

Volume 125, Number 5, May 1997, Pages 1309-1313

S 0002-9939(97)03802-1

\title{
$K$-REGULAR WITT RINGS
}

\author{
ROBERT W. FITZGERALD
}

(Communicated by Lance W. Small)

Abstract. We improve Kula's bounds on the size of possible $k$-regular Witt rings.

$(R, G, q)$ will denote an abstract Witt ring in the sense of [4]. Nearly all examples of interest are Witt rings of non-singular quadratic forms over a field of characteristic not two; however, using abstract Witt rings does simplify some proofs. The Witt ring is $k$-regular if there exists a 2-power $k$ such that for all $1 \neq x \in G$ we have $|D(1,-x\rangle|=k$. Such Witt rings were first studied in [1] primarily because the block design counting arguments there were perfectly suited to $k$-regular rings. However they remain unclassified.

We will always assume that $G$ is finite; set $g=|G|$. If $k=g$, then $R$ is totally degenerate and so classified by [4]. If $k=g / 2$, then $R$ is of local type [2] which is again classified in [4]. If $k=2$, then $R$ is a group ring extension of $\mathbb{Z}_{2}$ or $\mathbb{Z}_{4}$. If $2<k<g / 2$, then $R$ is not of elementary type and no examples are known or even expected. We will always assume that $2<k<g / 2$ and call such $k$-regular Witt rings exceptional.

It was shown in [1] that exceptional $k$-regular Witt rings satisfy $8 \leq k$ and $2 k^{2} \leq g$. Kula [3] improved both bounds and added an upper bound, showing:

$$
\begin{aligned}
16 \leq k, & \\
8 k^{2} \leq g \leq k^{4} / 4 & \text { if } k \equiv 1(\bmod 3), \\
8 k^{2} \leq g \leq k^{4} / 8 & \text { if } k \equiv 2(\bmod 3) .
\end{aligned}
$$

Here we show that $k^{3} \leq g$ and that if $k \equiv 1(\bmod 3)$ then $g \equiv 1(\bmod 3)$.

We fix some notation, which will agree with Kula's. $G^{*}$ denotes $G \backslash\{1\}$. We set $e=\log _{2} k$. For $a \in G^{*}$ and $i \geq 0$ set:

$$
X_{i}(a)=\left\{x \in G: x \neq 1, a \text { and }|Q(a) \cap Q(x)|=2^{i}\right\},
$$

where $Q(x)=\{q(x, y): y \in G\}$. Now for $x \neq a$,

$$
|Q(a) \cap Q(x)|=|D\langle 1,-a x\rangle| /|D\langle 1-a\rangle \cap D\langle 1,-x\rangle|=k /|D\langle 1,-a\rangle \cap D\langle 1,-x\rangle| .
$$

Thus we also have that:

$$
X_{i}(a)=\left\{x \in G: x \neq 1, a \text { and }|D\langle 1,-a\rangle \cap D\langle 1,-x\rangle|=2^{e-i}\right\} .
$$

Received by the editors November 21, 1995.

1991 Mathematics Subject Classification. Primary 11E81.

Key words and phrases. Witt ring. 
In particular, we may assume $0 \leq i \leq e$. We further set $n_{i}(a)=\left|X_{i}(a)\right|$ and write $X(a)$ for $X_{e}(a)$. For a 2-fold Pfister form $\rho$ we let $\rho^{\prime}$ denote the pure part of $\rho$.

We will use various equations derived by Kula:

$$
\begin{gathered}
\sum_{i=0}^{e-1}\left(2^{e-i}-1\right) n_{i}(a)=k^{2}-3 k+2, \\
g+\sum_{1 \neq \rho \in Q(a)}\left|D\left(\rho^{\prime}\right)\right|=1+\frac{g}{k}+\sum_{i=0}^{e} 2^{i} n_{i}(a), \\
|X(a) \cap X(b)| \geq g-2 k^{2}+6 k-7 \geq g-2 k^{2},
\end{gathered}
$$

where $a \neq b$ in $G^{*}$ for (3). Equation (1) is [3, 4.3b], (2) is equation (4.5.2) in [3, p. 45] and the first inequality of (3) is equation (4.3.1) in [3, p. 43]. The second inequality of (3) follows from our assumption that $k>2$.

We will also use two simple equations:

$$
\begin{gathered}
\sum_{i=0}^{e} n_{i}(a)=g-2, \\
\left|D\left(\rho^{\prime}\right)\right|<k^{2} \quad(\text { if } \rho \neq 1) .
\end{gathered}
$$

Both (4) and (5) appear in [3] but direct proofs are quick. (4) follows from $G \backslash\{1, a\}$ being the union of the $X_{i}(a)$. For (5), suppose $\rho^{\prime}=\langle a, b, a b\rangle$. Then

$$
D\left(\rho^{\prime}\right)=a \cdot \bigcup_{x \in D\langle 1, a\rangle} D\langle 1, b x\rangle .
$$

Since 1 occurs in each $D\langle 1, b x\rangle$ we have that $\left|D\left(\rho^{\prime}\right)\right|<|D\langle 1, a\rangle| \cdot k=k^{2}$.

Using equation (4) to find $n_{e}(a)$ and equation (1) to find $n_{e-1}(a)$, equation (2) may be re-written (see [3, pp. 45-46]) as:

$$
\begin{aligned}
g+\sum_{1 \neq \rho \in Q(a)}\left|D\left(\rho^{\prime}\right)\right|= & +\frac{g}{k}+g k-\frac{k^{3}}{2}+\frac{3 k^{2}}{2}-3 k \\
& +\sum_{i=0}^{e-2} 2^{i}\left(2^{e-i-1}-1\right)\left(2^{e-i}-1\right) n_{i}(a) .
\end{aligned}
$$

Proposition 1. If $k \equiv 1(\bmod 3)$, then $g \equiv 1(\bmod 3)$.

Proof. We may pick an $a \in G^{*}$ with $\langle\langle 1,1\rangle\rangle \notin Q(a) \backslash\{1\}$ (otherwise $-G^{*} \subset$ $D\langle 1,1,1\rangle$ while $|D\langle 1,1,1\rangle|<k^{2}$ by (5) and $\left|G^{*}\right| \geq 8 k^{2}-1$ by [3, 4.4]). Then for each anisotropic $\rho \in Q(a)$ we have that $\left|D\left(\rho^{\prime}\right)\right| \equiv 0(\bmod 3)$ by [3, 2.9]. Also, since for each $i$, in equation (6) one of $e-i-1$ or $e-i$ is even, we have that one of $2^{e-i-1}-1$ or $2^{e-i}-1$ is divisible by 3 . Assuming $k \equiv 1(\bmod 3)$, equation $(6)$ gives:

$$
g \equiv g+1+g-2(\bmod 3)
$$

and so $1 \equiv g(\bmod 3)$.

Theorem 1. $g \geq k^{3}$. 
Proof. Suppose there exists an exceptional $k$-regular Witt ring $(R, G)$ with $g<k^{3}$. Among all such Witt rings, choose one with minimal $h \equiv g / k^{2}$. Let $a$ and $b$ be distinct elements of $G^{*}$. Choose $x \in X(a) \cap X(b)$, which is possible by equation (3) and the fact that $g \geq 8 k^{2}[3,4.4]$. We use the equation (4.3.2) from [3, p. 43]:

$$
\begin{aligned}
h k=g / k=|Q(x)| & \geq|(Q(x) \cap Q(a))(Q(x) \cap Q(b))| \\
& =\frac{k^{2}}{|Q(x) \cap Q(a) \cap Q(b)|} \\
& \geq \frac{k^{2}}{|Q(a) \cap Q(b)|}=k|D\langle 1,-a\rangle \cap D\langle 1,-b\rangle| .
\end{aligned}
$$

A simple consequence of $(7)$ is that $|D\langle 1,-a\rangle \cap D\langle 1,-b\rangle| \leq h$. Pick a minimal $s \geq 0$ so that there exist distinct $a$ and $b$ in $G^{*}$ with $|D\langle 1,-a\rangle \cap D\langle 1,-b\rangle|=h / 2^{s}$. Set $2^{t}=|Q(a) \cap Q(b)|$. Then we have:

$$
g \geq 2^{s+2} k^{2} \text { and } t-s \geq 1 .
$$

Namely, if the first inequality failed, then $h=g / k^{2} \leq 2^{s+1}$. But then $\mid D\langle 1,-a\rangle \cap$ $D\langle 1,-b\rangle \mid \leq 2$ for all distinct $a$ and $b$ in $G^{*}$, while as noted in the first sentence of $\left[3\right.$, p. 44] we can always find distinct $a$ and $b$ in $G^{*}$ with $|D\langle 1,-a\rangle \cap D\langle 1,-b\rangle| \geq 4$. For the second inequality of (8) note that:

$$
2^{t}=|Q(a) \cap Q(b)|=\frac{k}{|D\langle 1,-a\rangle \cap D\langle 1-b\rangle|}=\frac{2^{s} k}{h} .
$$

Thus $2^{t-s} h=k$. By the assumption that $g=h k^{2}<k^{3}$ we have $2 h \leq k$ and so $t-s \geq 1$.

For each $x \in X(a) \cap X(b)$ we can rewrite (7) as:

$$
h k \geq \frac{k^{2}}{|Q(x) \cap Q(a) \cap Q(b)|} \geq \frac{k^{2}}{|Q(a) \cap Q(b)|}=\frac{h k}{2^{s}} .
$$

Then

$$
|Q(x) \cap Q(a) \cap Q(b)| \geq 2^{t-s}
$$

since otherwise $|Q(x) \cap Q(a) \cap Q(b)|<2^{t-s}=|Q(a) \cap Q(b)| / 2^{s}$ and equation (9) becomes:

$$
h k \geq \frac{k^{2}}{|Q(x) \cap Q(a) \cap Q(b)|}>\frac{2^{s} k^{2}}{|Q(a) \cap Q(b)|}=h k .
$$

List the elements of $Q(a) \cap Q(b)$ as $1, \rho_{2}, \ldots, \rho_{2^{t}}$. We have for each $x \in X(a) \cap$ $X(b)$ that $2^{t-s}-1$ of the $\rho_{i}$ 's lie in $Q(x)$, or equivalently, satisfy $-x \in D\left(\rho_{i}^{\prime}\right)$. Set $T_{x}$ equal to the number of $i$ 's, $2 \leq i \leq 2^{t}$, such that $-x \in D\left(\rho_{i}^{\prime}\right)$. Then:

$$
\sum_{x \in X(a) \cap X(b)} T_{x} \geq\left(2^{t-s}-1\right)|X(a) \cap X(b)| .
$$

Now this sum counts the number of pairs $(i, x)$ with $2 \leq i \leq 2^{t}, x \in X(a) \cap X(b)$ and $-x \in D\left(\rho_{i}^{\prime}\right)$. We can also count the number of such pairs by first fixing $i$. Namely:

$$
\sum_{x \in X(a) \cap X(b)} T_{x}=\sum_{i=2}^{2^{t}}\left|D\left(\rho_{i}^{\prime}\right) \cap-(X(a) \cap X(b))\right| .
$$


Now (11) implies that there exists an $i, 2 \leq i \leq 2^{t}$, such that:

$$
\left|D\left(\rho_{i}^{\prime}\right) \cap-(X(a) \cap X(b))\right| \geq \frac{1}{2^{t}-1} \sum_{x \in X(a) \cap X(b)} T_{x}
$$

and hence when combined with (9):

$$
\left|D\left(\rho_{i}^{\prime}\right)\right| \geq \frac{2^{t-s}-1}{2^{t}-1}|X(a) \cap X(b)| .
$$

Applying equations (5) and (3) yields:

$$
k^{2}>\frac{2^{t-s}-1}{2^{t}-1}\left(g-2 k^{2}\right) .
$$

If $s=0$, then (12) becomes $k^{2}>g-2 k^{2}$ which is impossible as $g \geq 8 k^{2}[3,4.4]$. Suppose then that $s \geq 1$. (12) is then:

$$
\left(2^{t}+2^{t-s+1}-3\right) k^{2}>\left(2^{t-s}-1\right) g .
$$

Use $g \geq 2^{s+2} k^{2}$ from the first part of (8) to get:

$$
\begin{gathered}
\left(2^{t}+2^{t-s+1}-3\right) k^{2}>\left(2^{t+2}-2^{s+2}\right) k^{2} \\
2^{s+2}+2^{t-s+1}-3>3 \cdot 2^{t} .
\end{gathered}
$$

Lastly, using $t-1 \geq s$ from the second part of (8) gives:

$$
\begin{gathered}
2^{t+1}+2^{t-s+1}-3>3 \cdot 2^{t}, \\
2^{t-s+1}-3>2^{t},
\end{gathered}
$$

which is impossible for $s \geq 1$. This contradiction shows $g \geq k^{3}$.

We combine these results with Kula's upper bound on $g$ and bound on $k$.

Corollary 1. For an exceptional $k$-regular Witt ring $(R, G)$ with $g=|G|: k \geq 16$ and

(1) if $k \equiv 1(\bmod 3)$, then $g \equiv 1(\bmod 3)$ and

$$
k^{3} \leq g \leq \frac{1}{4} k^{4},
$$

(2) if $k \equiv 2(\bmod 3)$, then

$$
k^{3} \leq g \leq \frac{1}{8} k^{4} .
$$

We note that the first open case is $k=16$ and $g=16^{3}=4096$.

Kula has shown that an exceptional $k$-regular Witt ring is non-formally real [3, Remark, p. 41] so that $I^{n} R=0$ for some $n$. We have:

Corollary 2. If $(R, G)$ is an exceptional $k$-regular Witt ring, then $I^{3} R \neq 0$. In fact, for any anisotropic 2-fold Pfister form $\rho, D(\rho) \neq G$.

Proof. $D(\rho)=\bigcup_{b \in D\left(\rho^{\prime}\right)} D\langle 1, b\rangle$ so that $|D(\rho)| \leq k\left|D\left(\rho^{\prime}\right)\right|<k^{3}$ by equation (5). Thus $D(\rho) \neq G$ by Theorem 1 . 


\section{REFERENCES}

1. R. Fitzgerald and J. Yucas, Combinatorial techniques and abstract Witt rings I, J. Algebra 114 (1988), 40-52. MR 89c:11058

2. I. Kaplansky, Fröhlich's local quadratic forms, J. Reine Angew. Math. 239 (1969), 74-77. MR 41:1644

3. M. Kula, Finitely Generated Witt Rings, Uniwersytet Ślaski, Katowice, 1991. MR 93b:11049

4. M. Marshall, Abstract Witt Rings, Queen's Papers in Pure and Applied Math., No. 57, Queen's University, Kingston, Ontario, 1980. MR 84b:10032

Department of Mathematics, Southern Illinois University, Carbondale, Illinois 62901-4408

E-mail address: rfitzg@math.siu.edu 